\title{
High Resolution Imaging in the Field Emission Scanning Electron Microscope at Low Accelerating Voltage and with Energy-Filtration of the Electron Signals
}

\author{
Raynald Gauvin ${ }^{1}$, Nicolas Brodusch ${ }^{1}$, Hendrix Demers ${ }^{1}$ and Patrick Woo ${ }^{2}$ \\ 1. Department of Mining and Materials Engineering, McGill University, Montreal, Quebec, Canada. \\ 2. Hitachi High-Technologies Canada Inc., Toronto, Canada.
}

For developing new technologies, it is important to characterize the microstructure of materials with high spatial resolution at the nanoscale. To achieve high resolution, field emission scanning electron microscopes (FE-SEM) were developed. These microscopes allow working at low accelerating voltage, below $5 \mathrm{kV}$, to take advantage of the reduction of the interaction volume with accelerating voltage (from $1 \mu \mathrm{m}$ in $\mathrm{Al}$ at $10 \mathrm{kV}$ to $10 \mathrm{~nm}$ at $1 \mathrm{kV}$ ). Furthermore, their higher gun brightness compared to conventional thermo-electronic emitters [1], allow a probe size at the nanoscale. However, technical problems arise when SEM operates at low $\mathrm{kV}$, i.e., the source brightness decreases and the chromatic aberration increases, all SEM parameters being equal. Using deceleration mode minimizes these problems and further improvement is achieved by using a cold-field emitter, which has a smaller energy spread and providing the highest brightness and the smallest source size of a FE-SEM. O.C. Wells proposed another way to attain higher resolution by separating the high-resolution signals from the lowresolution signals during the detection [2-3]. At low accelerating voltage, the emission volume of backscatter (BSE) and secondary ( $\mathrm{SE}_{\mathrm{II}}$, emitted by BSEs) electrons signals approach that of $\mathrm{SE}_{\mathrm{I}}$ (emitted by the primary electrons) signals. However it is not enough to reach the highest resolution. The use of a magnetic field above the sample and probe deceleration allows the improvement of the spatial resolution by collecting mostly high-resolution signals. In addition, the energy-filtration of the electron signals allows selecting the type of contrast detected: surface topography, compositional, or crystallographic.

Examples of high spatial resolution imaging are shown in Figure 1. A resolution of $2.5 \mathrm{~nm}$ (as calculated with SMART-J [4-5]) was obtained for a combination of SE and BSE signals in deceleration mode with a surface topography contrast (Figure 1A). Simultaneously, an energy-filtered BSE signal was acquired with a resolution of $1.9 \mathrm{~nm}$ and a compositional contrast was observed. Surface topography imaging at a very low accelerating voltage of $50 \mathrm{~V}$ is possible with the deceleration mode with still an excellent spatial resolution of $2.8 \mathrm{~nm}$. The energy-filtration allows the observation of small compositional contrast as shown in Figure 1D where $\mathrm{Al}_{3} \mathrm{Li}$ precipitates $(\delta$ ') were observed in a AA2099 Al-Li-Cu alloy. Monte Carlo simulations were used to understand and to optimize the SEM parameters of these different imaging modes. Figure 2 shows how the accelerating voltage and the range of energyfiltration affect the number of BSEs detected and their maximum exit position, which affect the spatial resolution.

The importance of Oliver C. Wells work on the understanding and development of improved electron detection modes cannot be overlooked. His legacy has directly influenced the development of modern SEM. The HITACHI SU-8230 CFE-SEM provides low accelerating voltage, deceleration mode and energy-filtration of the electron signals and thus allows the characterization of the microstructure of materials with high spatial resolution at the nanoscale with various types of contrasts. The development of these new technologies permits to extend the imaging capabilities of the SEM towards new nanoscale applications. 


\section{Reference:}

[1] J.I. Goldstein et al, "Scanning Electron Microscopy and X-Ray Microanalysis", (Springer, 3rd edition, 2003).

[2] O.C. Wells, "The construction of a scanning electron microscope and its application to the study of fibres", Ph.D. Dissertation, (Cambridge University, England, 1957).

[3] O. C. Wells, Scanning, 2 (1979), pp. 199-216.

[4] D.C. Joy, Journal of Microscopy, 208 (2002), pp. 24-34.

[5] J. Kim et al, Journal of Vacuum Science Technology B, 25 (2007), pp. 1771-1775.
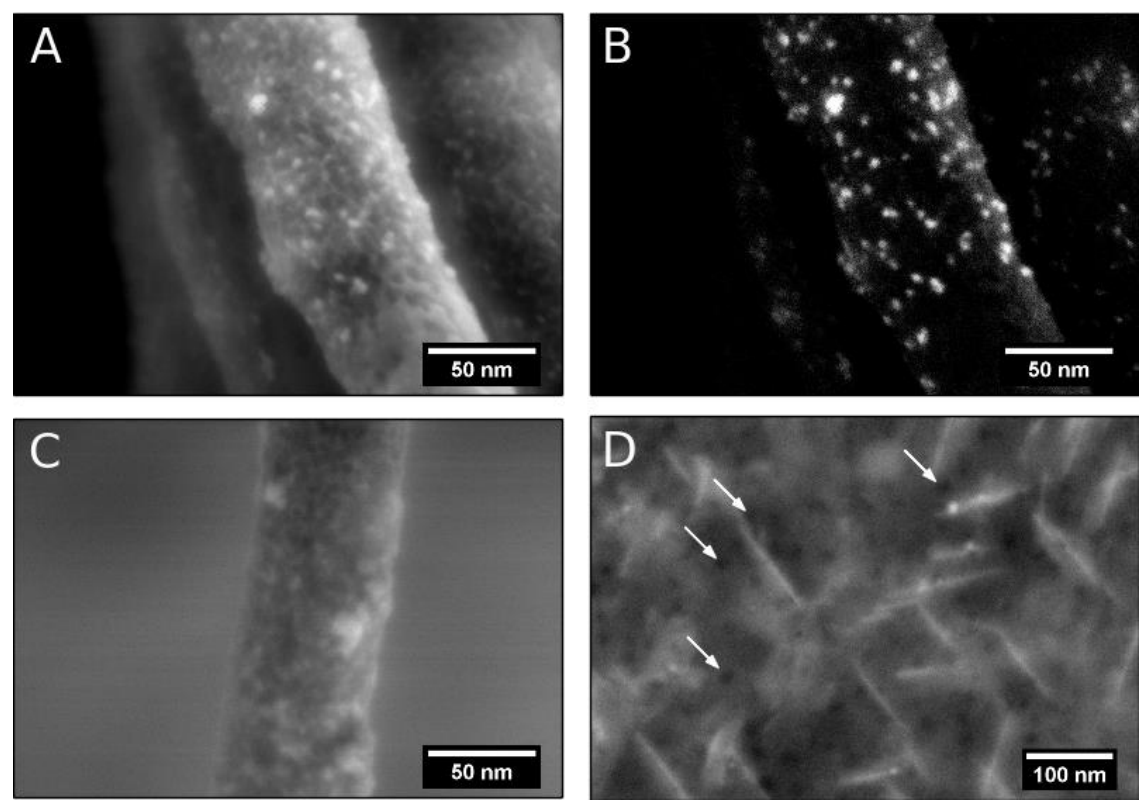

Figure 1. High resolution micrograph obtained with a cold-field emission scanning electron microscope. Carbon nanotubes (CNTs) decorated with platinum (Pt) nanoparticles micrographs were acquired at 1 $\mathrm{kV}$ in deceleration mode with: (A) combination of secondary and backscattered electron (SE+BSE) signals by the upper detector; (B) energy-filtered BSE signal by the top detector. (C) SE micrograph of a CNT-Pt was acquired at very low acceleration voltage $(50 \mathrm{~V})$ in deceleration mode with the top detector. (D) Energy-filtered BSE micrograph of a AA2099 aluminium-lithium alloy acquired at low energy with the upper detector. The AI3L1 precipitates $(\delta$ ') were observed (white arrows).
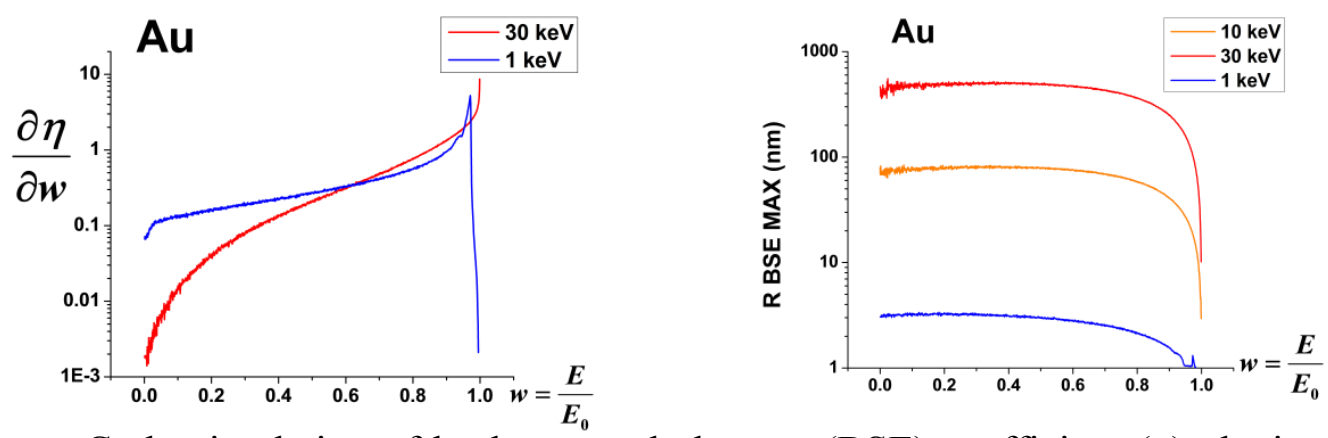

Figure 2. Monte Carlo simulation of backscattered electron (BSE) coefficient $(\eta)$ obtained for a gold bulk sample at different accelerating voltages $\left(E_{0}\right)$. Left, energy distribution of BSE $\partial \eta / \partial w$ versus BSE energy fraction $\left(w=E / E_{0}\right)$ for accelerating voltages of 1 and $30 \mathrm{kV}$. Right, maximum exit radius of BSE (R BSE MAX) versus energy fraction $(w)$ for accelerating voltages of 1,10 , and $30 \mathrm{kV}$. 